\title{
Personalisation of data-driven storytelling
}

\author{
Sebastian Meier ${ }^{\mathrm{a},}$, Fabian Dinklage $^{\mathrm{b}}$, Katrin Glinka ${ }^{\mathrm{c}}$ \\ aVISLAB.io,contact@sebastianmeier.eu \\ ${ }^{b}$ mail@fabiandinklage.com \\ ${ }^{c}$ Freie Universität Berlin, katrin.glinka@fu-berlin.de \\ * Corresponding author
}

Keywords: storytelling, personalisation, customisation, web cartography

\section{Introduction:}

Data-driven storytelling has become a common form of mass communication on the web for news outlets, science communicators and others. Actors form domains such as data journalism, cartography, or web development produce stories in a variety of forms and on a diverse range of topics. Many of such data-driven stories also use spatial data to, for example, highlight spatial patterns and trends. Some of these stories offer interactive elements, e.g., spatial- and temporalfiltering or details-on-demand, whereas the implementation of customisation or rather personalisation features is still quite rare. Even though personalisation features in data-driven storytelling are more complex to implement, they have the potential to offer readers personalised guidance in consuming the information, as well as to highlight the individual relevance of complex phenomena and their impact on the reader's life and lifeworld.
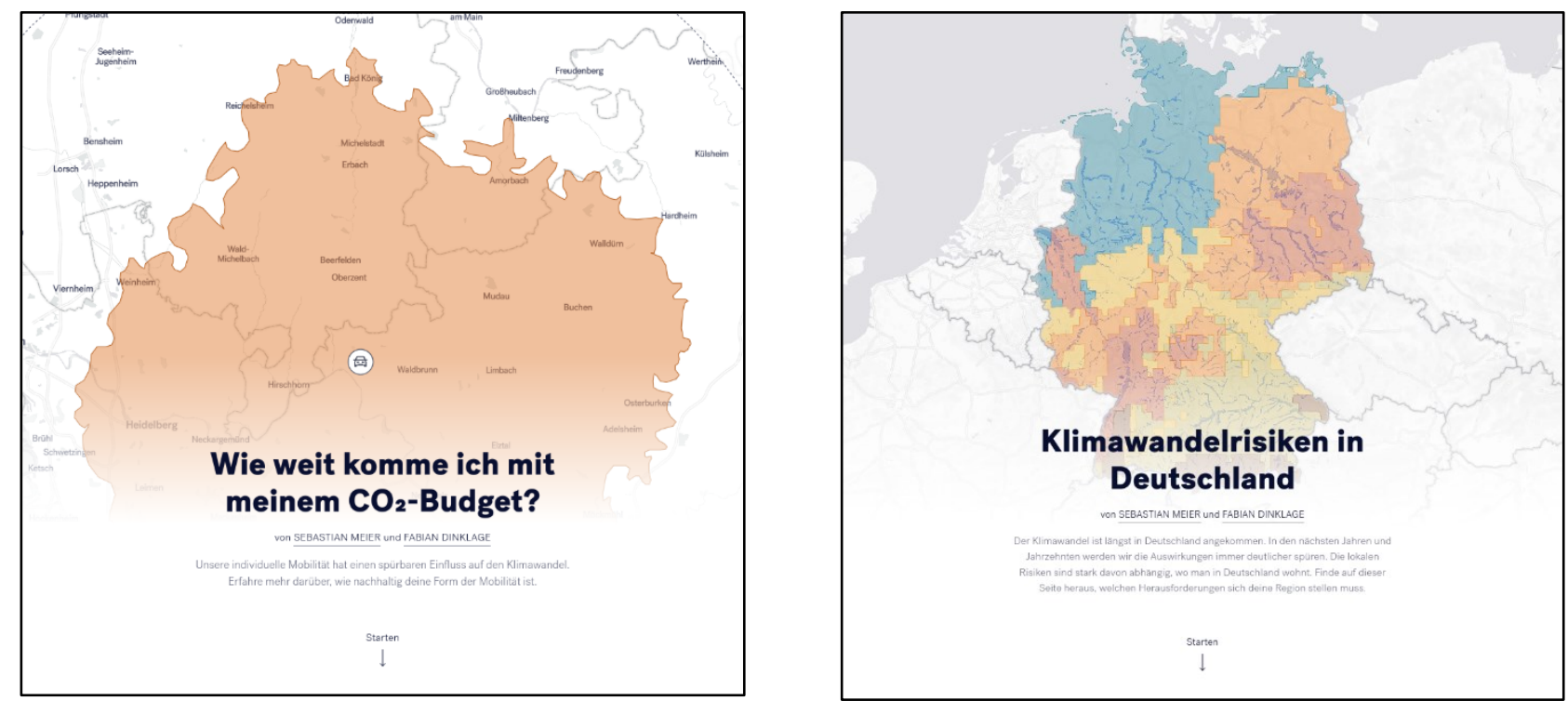

Figure 1 (left): Story on the need for Co2-reduction in Germany and the impact that the personal choice of mobility has on one's carbon footprint (co2mobilitaet.vislab.io); Figure 2 (right): Story on local climate change risks in Germany (klima-risiken.vislab.io)

As part of the research project "Location- and Context-based Storytelling and Surveys" (LoCobSS) we set out to explore new techniques and approaches for personalisation in data-driven storytelling in two case studies. Both address the challenge of communicating particularly abstract concepts like climate change. Climate change is a global long-term process, which makes the topic very abstract and often hard to relate to one's individual life in the present. To overcome this communication challenge, we explored techniques for personalisation in data-driven storytelling in order to highlight the impact of global climate change processes on a local and individual level. To demonstrate an approach for such personalisation techniques, we developed two exemplary case studies (see Fig. 1 \& 2) to highlight the conceptual and technical process. One case focuses on the need for Co2-reduction in Germany and the impact that the personal choice of mobility has on individual carbon footprint. The other case communicates local climate change risks in Germany focused on the users' location input (e.g. current location or region of interest).

\section{Related Works:}

The two case studies were informed by related works from the domain of personalisation (also referred to as customisation or adaption) of digital storytelling applications, as well as related approaches in information visualisation, GIS and map applications alike. Research in the domain of interactive storytelling often focuses on games (see e.g. Riedl \& Bulitko, 2013 and Yu \& Riedl, 2014) or, in connection with location based services, on museum or tourist applications (see e.g. 
Roussou \& Katifori, 2018 and Pujol et al, 2013). Personalisation in digital storytelling mostly refers to the generation or selection of one storyline from a multitude of possible combinations, based on e.g. user interactions or a user profile. While those approaches generate a variety of user-generated paths through a network of story points (content), it is still very "authored", since the story elements themselves are static and only the selection of a path depends on the user.

Other lines of research on data-driven storytelling include projects in which information visualisation projects make use of storytelling techniques in order to better convey relevant aspects of a data set (see e.g. Segel \& Heer, 2010; Rodríguez et al, 2015; Boy et al, 2015; Riche et al, 2018). Although data-driven techniques are being combined with storytelling approaches in these examples, they usually lack the personalisation aspect of the previous field.

One example for the combination of data-driven storytelling with personalisation is a project by Concannon et al. (2020). Here, a video story is generated based on the location of the user and the content of the video is modified based on the user input. Similar approaches to Concannon et al.'s modification of video content have long been used in map applications (see e.g. Aissi \& Gouider, 2012; Wilson et al 2010). In this case, the cartographic output is being modified based on user input or user preferences.

With our two use cases, we propose a conceptual and technical solution for combining those three elements: 1) storyline generation, 2) data-driven storytelling and 3) adaption of content, particularly thematic maps.

\section{Technique}

Conceptually, our approach to personalisation is based upon data spaces: the data space of the user and the data space of the story. To create a personal perspective on the story's data space, the user model is located within the story space. The term "space", in this context, is understood quite literally as in Tobler's first law of geography: "everything is related to everything else, but near things are more related than distant things" (Tobler 1970). By locating the user in the story space, we can highlight things "closer" and thereby potentially more relevant to the user (see also Meier \& Glinka 2017).

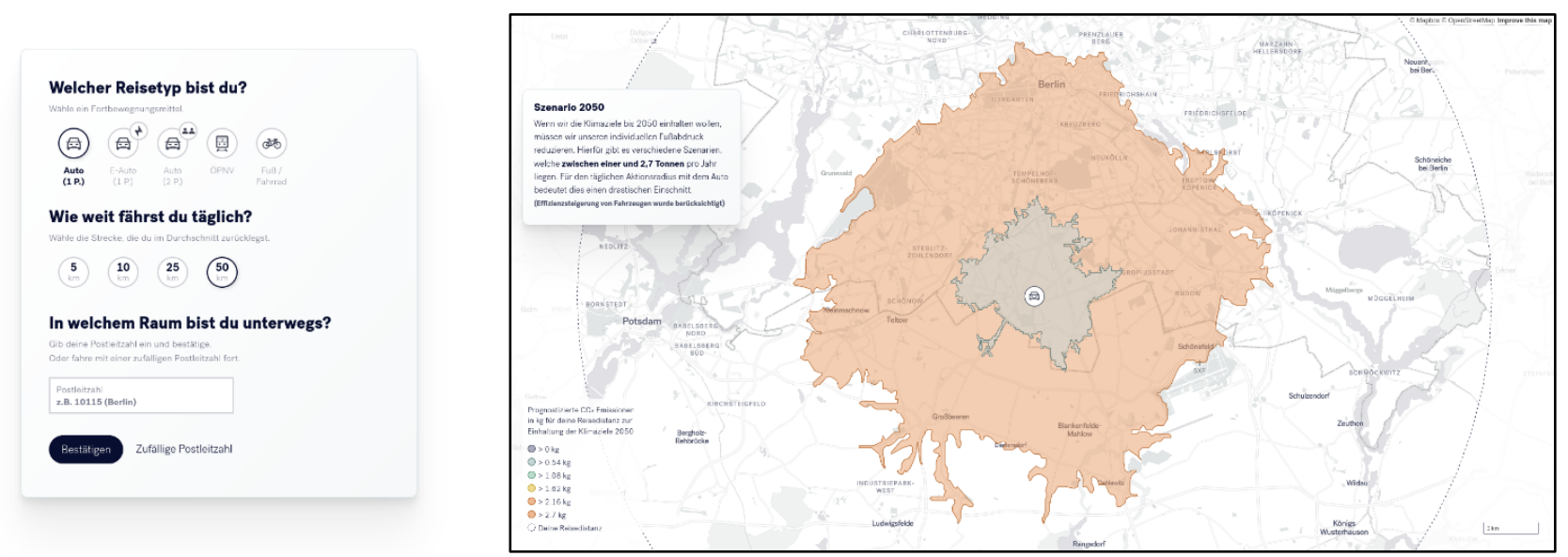

Figure 3 (left): Interface to collect information on the user to generate the user model to build the storytelling model upon. Figure 4 (right): Resulting visualisation based on the generated user model.

To construct the user model, we first need to acquire the user's features (see Fig. 3). As our focus is on spatial data-driven storytelling, the user location is an essential factor. To preserve the user's privacy and also to simplify the modelling, we are not using an exact location (latitude/longitude), but instead only the postcode that the users manually provide (e.g. current place of residence). Further features are being collected to refine the user model and further personalise the story (mode of transport, daily travel distance). Based on the user input, the constructed user model is referenced with the global "story data space". The combination of the two data spaces results in a customised data set. Based on the dataset, the storyline (path), which communicates the story from the perspective of the user, is being generated (see Fig. 5).

$\begin{array}{llll}\text { Model building } & \text { Data Space } & \text { Story Generation } & \text { Visualisation } \\ \text { Acquire user features: } & \text { Query data space } & \text { Generate story based } & \text { Generate visualisations based } \\ \text { (e.g. location, attributes, etc.) } & \text { with the user's model } & \text { on resulting data space } & \text { on data space and storyline }\end{array}$

Figure 5: Personalisation process.

\section{Exemplary case studies}

In the story on mobility and co2-reduction, for example, we collect information on location (postcode), primary mobility mode and daily travel distance. From the location input we furthermore derive the region type (rural, suburban, urban, metropolis). In our story data space, we subsequently reference those attributes, allowing us to contextualise the individual's mobility patterns in this specific region type and relate them to German averages. Our intention, here, is to 
create a personalised entry point for communicating mobility patterns in Germany. The travel distances that can be reached with each mobility type are visualised as isochrones specifically calculated for the postcode the user entered (see Fig. 4). This provides a personalised perspective, in this case by connecting abstract mobility patterns to a spatial region known by the user and their personal spatial point of reference. From there on, this personalised cartographic representation is used to discuss future scenarios that highlight the impact of co2-reduction efforts on mobility patterns. The scenarios are all adapted to the user's primary mobility patterns that were entered by the user at the beginning of the story. In the case of the mobility story, the combination of features resulted in more than 40.000 different story lines and cartographic representations.

\section{Future Research}

The cases built upon prior conceptual work on the topic (Meier \& Glinka 2017, 2018) and were designed to help us investigate the potentials and limitations of the techniques, particularly through user studies, in future research. The two case studies act as real-world tests to see, first of all, if the concept works in general and, second of all, how it could be implemented before conducting further research. The two applications have been deployed on the web and received more than 75.000 page views in 6 weeks after deployment. We received positive responses through social media and email, particularly highlighting the personalisation feature that allows users to explore the impact of climate change in their personal everyday surroundings. As a next step, we plan to conduct user studies to gain more refined insights into the effect of personalisation in data-driven storytelling.

While the proposed personalisation approaches cannot be applied to every data-driven story, we see potentials for highlighting individual relevance and supporting knowledge construction. As visualisation techniques in mass communication steadily grow in complexity, we need to develop tools and techniques to help non-expert users to navigate and understand those visualisations. We see a potential in the personalisation of data-driven storytelling as being one solution to overcome this literacy gap and making data-driven storytelling more engaging and comprehensible.

\section{Acknowledgements:}

The research was funded by the German Federal Ministry of Education and Research (BMBF) as part of the research project "Location- and Context-based Storytelling and Surveys" (LoCobSS). The source code and technical concepts have been published under an open-source license on GitHub. For more information see the project documentation: https://github.com/sebastian-meier/locobss-documentation

\section{References}

Aissi, S., \& Gouider, M. S. (2012). Personalization in Geographic information systems: A survey. arXiv preprint arXiv:1208.0153.

Boy, J., Detienne, F., Fekete, J. (2015) Storytelling in Information Visualizations: Does It Engage Users to Explore Data?. In Proceedings of the 33rd Annual ACM Conference on Human Factors in Computing Systems(Seoul, Republic of Korea) (CHI '15). ACM, New York, NY, USA, 1449-1458

Concannon, S., Rajan, N., Shah, P., Smith, D., Ursu, M., \& Hook, J. (2020, April). Brooke leave home: Designing a personalized film to support public engagement with open data. In Proceedings of the 2020 CHI Conference on Human Factors in Computing Systems (pp. 1-14).

Lin, Sophia (2018) Explore and eat your way around town with Google Maps, Google Blog, Accessed 2021-08-16 https://www.blog.google/products/maps/explore-around-town-google-maps

Meier, S., Glinka, K. (2017) The Individual in the Data — the Aspect of Personal Relevance. i-com 2017; 16(3): pp. 247265

Meier, S., Glinka, K., (2018) Data-driven personal cartographic perspectives. Kartographische Nachrichten, (13/8)

Pujol, L., Katifori, A., Vayanou, M., Roussou, M., Karvounis, M., Kyriakidi, M., ... \& Ioannidis, Y. (2013). From personalization to adaptivity-Creating immersive visits through interactive digital storytelling at the Acropolis Museum. In Workshop proceedings of the 9th International conference on intelligent environments (pp. 541-554). IOS Press.

Riche, N.H., Hurter, C., Diakopoulos, N., \& Carpendale, S. (Eds.). (2018). Data-Driven Storytelling (1st ed.). A K Peters/CRC Press.

Riedl, M. O., \& Bulitko, V. (2013). Interactive narrative: An intelligent systems approach. Ai Magazine, 34(1), 67-67.

Rodríguez, M., Nunes, S., and Devezas, T. (2015) Telling Stories with Data Visualization. In Proceedings of the 2015 Workshop on Narrative \& Hypertext (Guzelyurt, Northern Cyprus) (NHT '15). ACM, New York, NY, USA, 7-11.

Roussou, M., \& Katifori, A. (2018). Flow, staging, wayfinding, personalization: Evaluating user experience with mobile museum narratives. Multimodal Technologies and Interaction, 2(2), 32. 
Segel, E. and Heer, J. (2010) Narrative visualization: Telling stories with data. IEEE transactions on visualization and computer graphics 16, 6(2010), 1139-1148.

Tobler, W. (1970) A computer movie simulating urban growth in the Detroit region. Economic Geography, 46: 234-40

Wilson, D., Bertolotto, M. \& Weakliam, J. (2010) Personalizing map content to improve task completion efficiency, International Journal of Geographical Information Science, 24:5, 741-760, DOI: 10.1080/13658810903074490

Yu, H. and Riedl, M. (2014) "Personalized Interactive Narratives via Sequential Recommendation of Plot Points," in IEEE Transactions on Computational Intelligence and AI in Games, vol. 6, no. 2, pp. 174-187, June 2014. 